\title{
Circumference Measurements in Obstetrical Ultrasound: Ellipse vs. Arithmetic Mean
}

\author{
Ronald S. Adler, PhD, MD, Richard A. Bowerman, MD, \\ and Jonathan M. Rubin, MD, PhD
}

Quantitation of fetal parameters by prenatal sonography enables the assessment of menstrual age and fetal development. Appropriate fetal growth can be surmised on the basis of sequential measurements, as well as the relative variation of parameters such as biparietal diameter (BPD), femur diaphysis length (FDL), abdominal circumference (AC), and head circumference (HC). Accurate determination of these quantities to within an acceptable range of measurement error is essential to ensure reproducibility of results and to document true growth patterns.

Assuming images are acquired at appropriate anatomic levels, major sources of measurement error are related to

(1) the ability to resolve end points of a linear dimension or to define an edge;

(2) inter- or intraobserver variation in performing a measurement; and

(3) anatomic molding, which may distort anatomy, as in the case of late gestation or with oligohydramnios.

For parameters such as $\mathrm{HC}$ and $\mathrm{AC}$, one must in addition consider the manner in which the circumference is determined. ${ }^{1}$ Such calculations may be performed at the time of scanning by an ellipse region-of-interest (ROI) built into the ultrasound unit, curve tracing that uses a tracker ball or joy stick, or by application of a simple equation following measurement of major and minor diameters of either head or abdomen. Let the quantities $2 a$ and $2 b$ denote major and minor diameters, respectively, and $C$ denote the circumference to be determined; then

$$
C \simeq \pi(a+b)
$$

From the Department of Radiology, University of Michigan Hospitals, Ann Arbor, Michigan. For reprints contact Ronald S. Adler, PhD, MD, Department of Radiology, Box 0030, University Hospital, B1 D502, 1500 East Medical Center Drive, Ann Arbor, Michigan 48109-0030.
Several authors ${ }^{2-4}$ have incorrectly taken Eq. (1) as well as other expressions to represent the circumference of an ellipse, although empirically determined $\mathrm{AC}$ and $\mathrm{HC}$ values would appear to agree fairly well with these approximations. The correct formula for an ellipse has since been stated within the context of obstetrical measurements ${ }^{5,6}$; however, the precise range over which Equation (1) is valid has not specified in general terms.

Equation (1) has the advantage of being a simple expression that is easily applied in a clinical setting. If an ellipse is, in fact, a reasonable approximation of the structure being measured, it is of interest to ascertain whether the calculated elliptical circumference represents a significant improvement over Equation (1) while maintaining clinical applicability. The alternative is to determine over what physiologic range of $a$ and $b$ the two expressions closely approximate one another. Significant differences would be important for reasons already mentioned.

In the following sections, the exact expression for the circumference of an ellipse is compared to Equation (1) as a function of $e=b / a$. For the range of values of $e$ normally seen in obstetrical ultrasound, it is demonstrated that the two expressions closely approximate one another. However, as $e \rightarrow 0$, the two circumference formulas may differ by as much as $\sim 25 \%$.

\section{ELLIPSE APPROXIMATION}

Let $\mathrm{C}_{E}$ denote the circumference of an ellipse obtained from major and minor axes $a$ and $b$, respectively. $C_{E}$ is then given by

$$
\begin{aligned}
C_{E} & =2 \pi a f(e) \\
e & =b / a
\end{aligned}
$$

$f(e)$ being the complete elliptic integral ${ }^{7}$ 


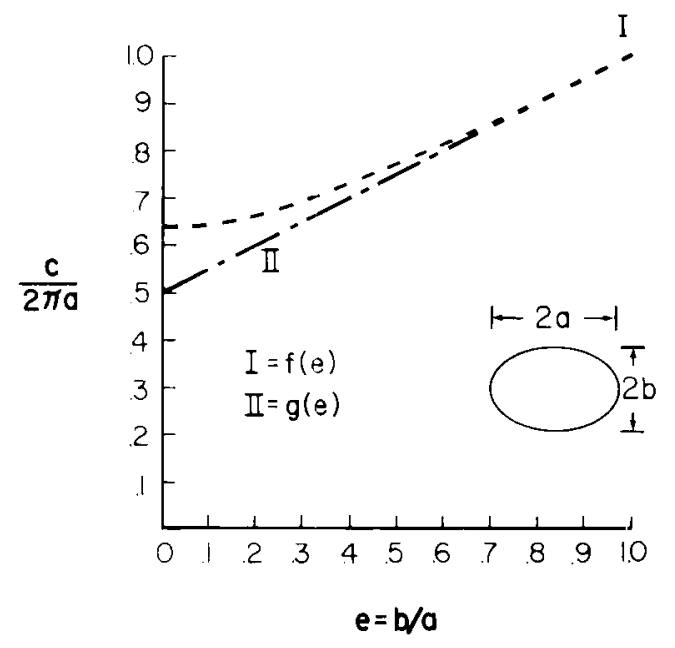

FIGURE 1. Distortion introduced by an ellipse is compared to that estimated by the two-circle approximation. An ellipse with major and minor radii, $a$ and $b$, respectively, is assumed. Even for values of $e$ as small as 0.6 , circumferences obtained from the two-circle approximation vs the expression for an ellipse agree to within $1 \%$ to $2 \%$.

$$
f(e)=\frac{2}{\pi} \int_{0}^{\pi / 2} d \Theta\left[1-\left(1-e^{2}\right) \sin ^{2} \Theta\right]^{1 / 2}
$$

Comparison of Equation (2) to the "two-circle" approximation [Equation (1)] is facilitated by defining a new function,

$$
g(e)=(1+e) / 2
$$

such that Equation (1) becomes

$$
C=2 \pi a g(e)
$$

Variation of $f(e)$ and $g(e)$ are depicted in Figure 1 ; note that for values of $e$ as small as $1 / 2$, these functions differ by no more than about $2.5 \%$. Inasmuch as $e \geq 2 / 3$ in most physiologic situations, Equation (1) serves as an excellent approximation to Equation (2a). As $b \rightarrow 0, C_{E} \rightarrow 4 a$, which is the correct expression for a rod of length $2 a$.

Precise interpretation of Equation (1) may be obtained when the minor diameter is determined at a sufficiently slowly varying convex boundary

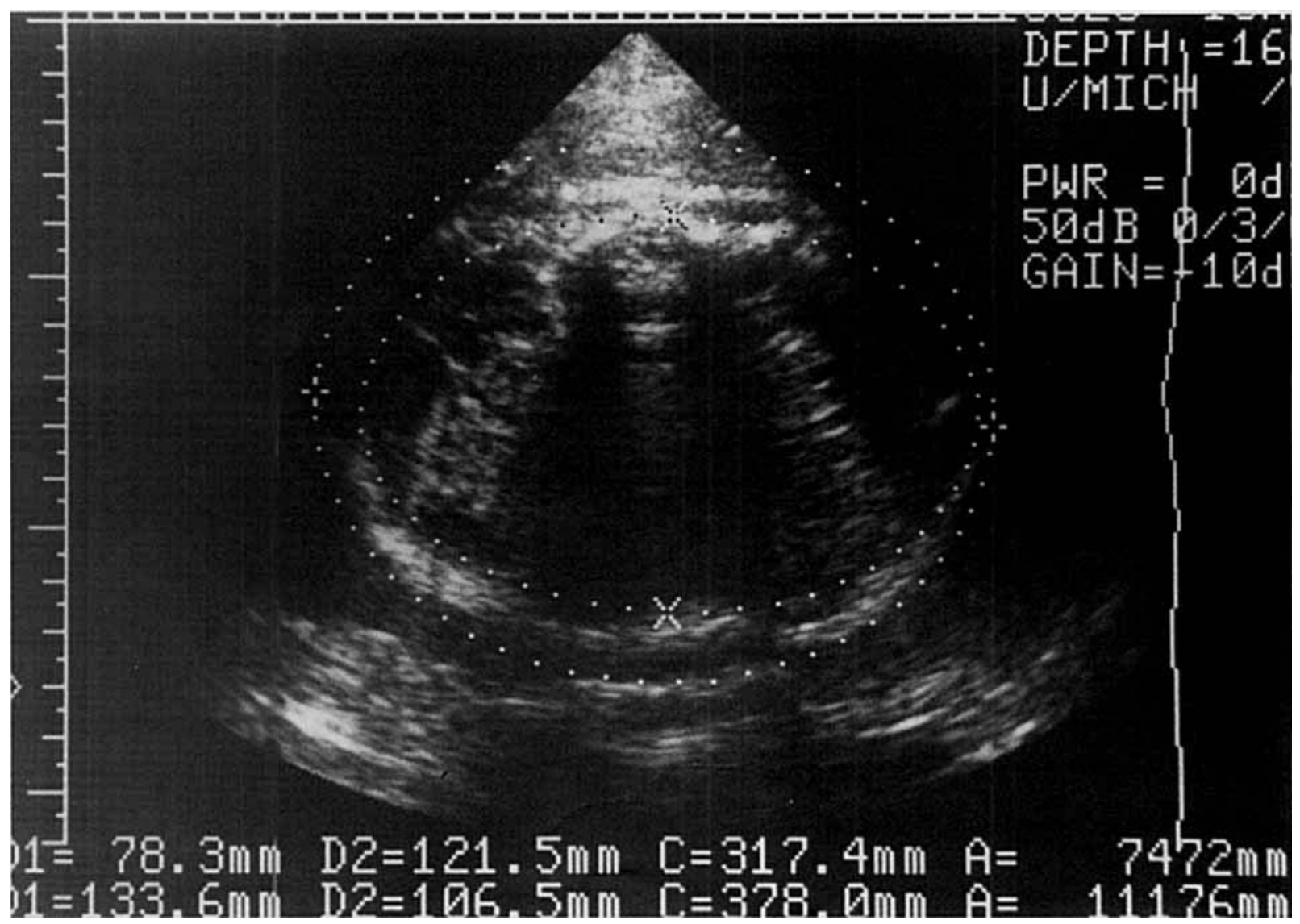

FIGURE 2. Absolute error estimated from two-circle approximation vs "tightly" circumscribed/inscribed ellipse. The above section is obtained through the fetal abdomen. The two circumferences are obtained from an ellipse. The above section is obtained through the fetal abdomen. The two circumferences are obtained from an ellipse ROI built into the ultrasound unit. Absolute error estimated from the ROl is approximately $3 \mathrm{~cm}$. The corresponding estimate from the two-circle approximation [Equation (6)] is $8.7 \mathrm{~cm}$. 
(i.e., radius of curvature $\geq b$ ). Let $C$ represent the circumference to be determined. Given the set of circles that circumscribe $C, 2 \pi a$ is a least upper bound. Alternatively, given the set of circles that inscribe $C, 2 \pi b$ is a greatest lower bound. Since both arithmetic mean, $2 \pi a$ g(e), and $C$ are bounded by these circles, we obtain the following inequality for the absolute error $E$,

$$
E=|C-2 \pi \mathrm{ag}(e)| \leq \pi|a-b|
$$

which is expected to reflect $E$ accurately only for $b \sim a$. The vertical bars, || , denote absolute value. A more percise determination could be obtained utilizing the "tighter fit" afforded by the ellipse ROI option (i.e., Figure 2).

\section{CONCLUSION}

The validity of the two-circle approximation [Equation (1)] has been tested empirically in infants just prior to delivery as compared to immediate postnatal measurements. ${ }^{2}$ Such comparisons evaluate the composite error introduced by both assumed geometry as well as measurement (i.e., edge detection, observer variation, etc.). Taking an ellipse as an accurate representation of either head or abdominal circumference, Equation (1) provides an excellent approximation to the actual circumference of the ellipse [Equation (2)] for the expected physiologic range of measured parameters. This approximation is expected to be applicable in cases of molding, dolichocephaly, or oligohydramnios. In situations in which a simple geometric interpretation to
Equation (1) applies, a least upper bound to the absolute error $E$ is obtained for the class of twocircle approximations [Equation (6)].

\section{REFERENCES}

1. Campell S, Thomas A: Ultrasound measurement of the fetal head to abdomen circumference ratio in the assessment of growth retardation. $\mathrm{Br} J$ Obstet Gynecol 84:165, 1977.

2. Crane JP, Kopta MM: Prediction of intrauterine growth retardation via ultrasonically measured head/abdominal circumference ratios. Obstet Gynecol 5415:597, 1979.

3. Hadlock FP, Kent WR, Loyd JL, et al: An evaluation of two methods of measuring fetal head and body circumferences. J Ultrasound Med 1:359, 1982.

4. Woo JSK, Liang St, Wan CW, et al: Abdominal circumference vs. abdominal area-Which is better? $J$ Ultrasound Med 3:101, 1984.

5. DeVore GR, Platt LD: Choosing the correct equation for computing the head circumference from two diameters: The effect of head shape. Am J Obstet Gynecol 148:221, 1984.

6. Toms, DA: The circumference of an ellipse: Letters to the editor. J. Ultrasound Med. Woo JSK, Hadlock FP: In reply to the letters to the editor. $J \mathrm{Ul}$ trasound Med 3:480, 1984.

7. Birnholz JC: On calculating the peremiter of an ellipse. J Clin Ultrasound 12:55, 1984.

8. Schwartz A: Calculus and Analytic Geometry (ed 2). Holt, Rhinehart and Winston, New York, 1967.

9. Tamura RK, Sabbagha RE, Pan W, Vaisrub N: Ultrasonic fetal abdominal circumference: Comparison of direct versus calculated measurement. Obstet Gynecol 67:833, 1986. 